\title{
Static and Dynamic Forces between Adsorbed Polyelectrolyte Layers (Quaternized Poly-4-vinylpyridine)
}

\author{
Marina Ruths, ${ }^{*}$, Svetlana A. Sukhishvili, ${ }^{\ddagger}$ and Steve Granick* \\ Department of Materials Science and Engineering, University of Illinois, Urbana-Champaign, \\ Urbana, Illinois 61801
}

Received: January 17, 2001; In Final Form: April 26, 2001

\begin{abstract}
Static forces (a combination of steric and electrostatic interactions) and dynamic shear forces (resulting from interactions between polymer chains) were measured between quaternized poly-4-vinylpyridine layers (QPVP) adsorbed onto mica from $1 \mathrm{mM}$ borate buffer with or without added $0.25 \mathrm{M} \mathrm{NaCl}$. At the polyelectrolyte concentration chosen, $0.4 \mathrm{mg} \mathrm{mL}^{-1}$, extended layers were formed at all adsorption conditions. The adsorbed amount of $98 \%$ quaternized poly-4-vinylpyridine was lower at low ionic strength than of $14 \%$ quaternized, and increased with added salt. Layers formed by one-step adsorption showed a predominantly elastic response to small-amplitude oscillatory shear in the frequency range $0.13-130 \mathrm{~Hz}$. In the case of two-step adsorption, the confined layers allowed viscous dissipation of similar magnitude as the elastic, which suggests that additional chains adsorbing at high salt concentration onto a preformed layer had fewer segments in contact with the solid surface.
\end{abstract}

\section{Introduction}

Polyelectrolytes (charged polymers) are commonly used to control the stability of colloidal dispersions in aqueous systems, for example in paper-making and in water-based paints. The adsorption of polyelectrolytes on surfaces, which has been studied extensively both experimentally ${ }^{1-20}$ and theoretically, ${ }^{21-31}$ is primarily the result of electrostatic attraction that leads to an entropically favored replacement of small, adsorbed counterions with charged segments of the macromolecule. It is also influenced by van der Waals forces. In this study, we will not be concerned with the specific lock-and-key interactions that are prominent in some biological systems. Due to desorption of hydrated small cations from many solid surfaces into aqueous solution, ${ }^{32}$ negatively charged surfaces are abundant in nature. The adsorption of cationic polyelectrolytes is therefore of large practical importance.

The different conformations of polyelectrolyte chains in solution and on a surface result from a balance between elastic (stretching) energy and conformational entropy. In addition to these usual considerations for neutral polymer chains, there are additional electrostatic interactions between segments. ${ }^{21-31}$ The details of how to describe this contribution have proven difficult and controversial to quantify. Typically, highly charged polyelectrolytes adsorbing at low concentration from low ionic strength solution form very flat layers on oppositely charged surfaces, $3,5-12,15,17-31$ and the adsorbed amount is not strongly sensitive to the chain length. In the absence of other interactions, an increased concentration of counterions would compete with the polyelectrolyte for adsorption sites and cause a decrease in the adsorbed amount. ${ }^{25}$ However, in the presence of van der Waals or specific surface attractions, the amount of highly

\footnotetext{
* Corresponding authors.

$\dagger$ Present address: Department of Physical Chemistry, Åbo Akademi University, Porthansgatan 3-5, FIN-20500 ̊̊bo, Finland.

$\doteqdot$ Present address: Department of Chemistry and Chemical Biology, Stevens Institute of Technology, Castle Point on Hudson, Hoboken, NJ 07030 .
}

charged polyelectrolyte adsorbed from low solution concentration initially increases with increasing salt concentration due to screening of the repulsion between segments, and forms a progressively more extended layer. ${ }^{3-7,9,11,12,18,21,24,25,29-33 ~ E x-~}$ perimentally, it has been found ${ }^{11,12,19,33}$ that the time needed for equilibration after changing the adsorption conditions can be very long due to the slow rearrangement of long chains with many strongly adsorbed segments. This leads to difficulties in determining the equilibrium conformation at different conditions.

One distinguishes between weak polyelectrolytes, where the charge density depends on the $\mathrm{pH}^{1,6-8,12,15,20,24,27,28}$ (dissociation of polyacids and polybases), and strong polyelectrolytes ${ }^{2-5,9-21,24,25}$ that contain permanent charges in aqueous solution. We have studied a strong polyelectrolyte system, $14 \%$ and $98 \%$ quaternized poly-4-vinylpyridine, adsorbed on mica from $0.4 \mathrm{mg} \mathrm{mL}^{-1}$ aqueous solution at high $\mathrm{pH}$ and varying electrolyte concentration. Our emphasis was (i) to compare (see (ii) later) layer thickness, extension, and interfacial rheology at different charge density, and (ii) to study effects of adsorption history when the layers were adsorbed from a higher solution concentration than in most previous studies of polyelectrolytes on mica. ${ }^{5-11,14,17,20}$ The adsorbed amount is compared to recent experiments on the adsorption of these polymers on oxidized silicon at similar conditions, ${ }^{18,19,33}$ and to a study on $98 \%$ quaternized poly-4vinylpyridine adsorbed on mica from 0.005 and $1.0 \mathrm{mg} \mathrm{mL}^{-1}$ solution. ${ }^{33}$ The interaction forces measured on approach and separation of two adsorbed layers are compared to studies on other strong cationic polyelectrolytes with high charge density at low concentration of added salt and significantly lower polyelectrolyte concentration than in our systems,,${ }^{5-11,17,20}$ and to studies of partially charged ones $\left(1 \%,{ }^{14} 10 \%,{ }^{13}\right.$ and $\left.30 \%{ }^{16}\right)$ at concentrations lower than or comparable to in our systems. Measurements of dynamic (shear) interactions as a function of separation distance were used to detect the extension of the adsorbed polyelectrolyte layers. The total electrolyte concentration and the order of adsorption from different solution conditions were found to strongly affect the adsorbed amount 
and the extension of the chains. The measurements of interaction forces as a function of distance together with the responses of strongly compressed layers to shear suggest that, on the timescale of our experiment, different conformations of the same polyelectrolyte result from different adsorption paths.

\section{Experimental Section}

The interaction forces and shear properties of two adsorbed polyelectrolyte layers in aqueous solution at $25{ }^{\circ} \mathrm{C}$ were measured using a surface forces apparatus (SFA), modeled after the "Mark II" SFA ${ }^{34}$ and equipped with a device for oscillatory lateral shear. ${ }^{35,36}$ The surfaces were thin sheets of muscovite mica, which were silvered on their backside and glued onto half-cylindrical, fused silica support disks with a thermosetting epoxy glue, EPON 1004F (Shell). The surface separation, D, relative to adhesive contact between the mica sheets in dry air, was measured by multiple beam interferometry with an accuracy of 1-2 $\AA^{34,37}$ The lower surface was supported on a double cantilever spring with a spring constant of $930 \mathrm{~N} / \mathrm{m}$, allowing the force between the surfaces to be determined by monitoring the spring deflection as a function of separation. At points where the gradient of the interaction force exceeds the spring constant, the surfaces will spontaneously jump to the next stable position.

The linear shear response of two approaching polyelectrolyte layers in solution was studied as a function of separation at a frequency of $f=1.3 \mathrm{~Hz}$ and a displacement amplitude of a few angstroms. Strongly compressed layers were also studied in small-amplitude shear experiments in a frequency range of $f=$ $0.13-130 \mathrm{~Hz}$, and at a constant frequency of $f=13 \mathrm{~Hz}$ with a displacement amplitude of $0.3-1900 \AA$. The shear was induced by applying a sinusoidal voltage to a piezoelectric bimorph element on a device holding the upper surface. The lateral motion is resisted by the viscoelastic fluid layer confined between the two surfaces, and the complex shear force can be calculated from the damping and phase shift of the oscillation as detected from the voltage induced in another, symmetrically placed bimorph element. The response can be separated in an elastic force (in phase with the drive) and a dissipative force (out of phase). The elastic and viscous force constants, $f_{\text {elastic }} / x$ and $f_{\text {viscous }} / x$ (where $x$ is the displacement amplitude in oscillatory shear deformation), can be normalized by the effective contact area multiplied by the film thickness to give effective elastic and viscous shear moduli, $G_{\text {eff }}^{\prime}$ and $G^{\prime \prime}$ eff. The contact area for the thinnest films (high compression) was calculated from the diameter of the flattened tip of the interference fringes. For thicker films, it was estimated from the "Langbein approximation" as described in ref 38 .

The polyelectrolyte was poly-4-vinylpyridine (Polymer Source, Quebec), quaternized to different degrees, $14 \%$ and $98 \%$ (determined by infrared spectroscopy), with ethyl bromide in ethanol solution. ${ }^{18}$ The number average molecular weight before quaternization was $M_{\mathrm{n}}=34200 \mathrm{~g} / \mathrm{mol}$ (degree of polymerization $n=325$ ) with a polydispersity of $M_{\mathrm{w}} / M_{\mathrm{n}}=1.23$, and after quaternization the $M_{\mathrm{n}}$ was $39000 \mathrm{~g} / \mathrm{mol}$ and $69000 \mathrm{~g} / \mathrm{mol}$ for the two degrees of quaternization, respectively. ${ }^{18}$

The adsorption was done outside the SFA by immersing support disks with mica in $0.4 \mathrm{mg} \mathrm{mL} \mathrm{m}^{-1}$ quaternized poly-4vinylpyridine (QPVP) solution at $\mathrm{pH}=9.2$ for $1-1.5 \mathrm{~h}$ at room temperature. The mica surface is negatively charged in the buffer solution, and positively charged QPVP segments adsorb to replace small adsorbed cations. In addition, both charged and uncharged segments interact with the surface through van der Waals forces. FTIR measurements suggest that on negatively charged silica surfaces, the adsorbed amount of QPVP reaches a constant value after ca. $50 \mathrm{~min}$ at a concentration of 0.005 $\mathrm{mg} \mathrm{mL} \mathrm{L}^{-1},{ }^{18}$ and that the adsorption is more rapid with increasing concentration (constant amount after $10 \mathrm{~min}$ at $0.1 \mathrm{mg} \mathrm{mL}^{-1}$ ). The solvent was either $1 \mathrm{mM}$ borate buffer (Borax, $\mathrm{Na}_{2} \mathrm{~B}_{4} \mathrm{O}_{7}$. $10 \mathrm{H}_{2} \mathrm{O}$, EM Science (Merck)) or $1 \mathrm{mM}$ borate buffer containing $0.25 \mathrm{M} \mathrm{NaCl}$. The water used to prepare the solutions was distilled and passed through a Barnstead Nanopure II deionizing and filtering system. After the adsorption, nonadsorbed polymer was removed by immersing the surfaces in borate buffer or buffer with $0.25 \mathrm{M} \mathrm{NaCl}$ (conditions matching the ones used for adsorption) for 30-40 min. In FTIR experiments on silicon oxide surfaces, no desorption of $48 \%$ QPVP was found when rinsing with buffer solution with similar ionic strength as the adsorption solution, ${ }^{19}$ nor did $98 \%$ QPVP desorb when the adsorption solution was replaced with 0.1 or $0.5 \mathrm{M} \mathrm{NaCl}$ solution. ${ }^{33}$ It is thus unlikely that the amount of adsorbed polymer decreases significantly during the rinsing in the present experiments. This is supported by other studies where a $10 \%$ charged copolymer was found not to desorb from mica in pure water within $12 \mathrm{~h},{ }^{12}$ and another, fully charged cationic polyelectrolyte was shown to desorb to a small extent only after immersion in dilute electrolyte solution for days. ${ }^{39}$

When removing the disks from the rinsing buffer, they were held so that a drop of the liquid remained on each surface while they were being mounted in the SFA, and a $0.2 \mathrm{~mL}$ drop of borate buffer (or buffer with $0.25 \mathrm{M} \mathrm{NaCl}$, according to the adsorption conditions) was immediately added to merge these opposed drops. As a result, the liquid between the QPVP layers during the experiment had a similar electrolyte concentration as the adsorption solution, but did not contain free polymer. (At a concentration of $0.4 \mathrm{mg} \mathrm{mL} \mathrm{m}^{-1}$ and assuming full dissociation, the 14\% and 98\% QPVP in the adsorption solutions contribute $0.5 \mathrm{mM}$ and $1.8 \mathrm{mM} \mathrm{1:1} \mathrm{electrolyte,} \mathrm{respectively,} \mathrm{to}$ the total ionic strength). A separate series of experiments were done as a two-step adsorption where 98\% QPVP was adsorbed first for $1 \mathrm{~h}$ from borate buffer, then for $1 \mathrm{~h}$ from borate buffer with $0.25 \mathrm{M} \mathrm{NaCl}$. These surfaces were then immersed in buffer with $0.25 \mathrm{M} \mathrm{NaCl}$ for $40 \mathrm{~min}$, mounted as above, and the measurements were done in buffer with $0.25 \mathrm{M} \mathrm{NaCl}$.

Since the atmosphere in the chamber of the instrument was exposed to $\mathrm{P}_{2} \mathrm{O}_{5}$ to protect the piezoelectric bimorph elements of the shear device from humidity, evaporation occurred of water from the drop between the surfaces. Each experiment was therefore started immediately after mounting the surfaces, and discontinued after $1.5-2 \mathrm{~h}$ when an evaporation of up to $30 \%$ of the drop could be seen. This change in volume affected the electrolyte concentration, mainly in the experiments were $\mathrm{NaCl}$ was used, but was too small to affect the $\mathrm{pH}$ of the buffer solution.

\section{Results and Discussion}

Adsorbed Amount at Different Charge Density and Salt Concentration. We have studied $14 \%$ and $98 \%$ QPVP layers adsorbed from a higher solution concentration $\left(0.4 \mathrm{mg} \mathrm{mL}^{-1}\right)$ than in most previous studies of polyelectrolytes on mica. ${ }^{5-11,14,17,20}$ The final thickness of two compressed layers ("hard-wall" separation) varied with the charge density and with the electrolyte concentration of the adsorption solution. The adsorbed amount on each surface calculated from this thickness (assuming a density of $1.15 \mathrm{~g} / \mathrm{cm}^{3}$ as for uncharged PVP, ${ }^{40}$ also for the values from ref 33) is given for each system in Table 1 together with values from other investigations. ${ }^{18,33}$ The compressed layers also contain solvent, so that the values given in Table 1 represent an upper bound for the adsorbed amount on 
TABLE 1: Adsorbed Amount of QPVP $\left(\mathrm{mg} / \mathrm{m}^{2}\right)$ from $1 \mathrm{mM}$ Borate Buffer $(\mathrm{pH}=9.2)$ at Different Conditions

\begin{tabular}{llccc}
\hline \multicolumn{1}{c}{ concentration; substrate; method } & 14\% QPVP & 98\% QPVP & 98\% QPVP, 0.25 M NaCl & $\begin{array}{r}\text { two steps: (1) 98\% QPVP } \\
(2) 98 \% \text { QPVP, 0.25 M NaCl }\end{array}$ \\
\hline $0.005 \mathrm{mg} \mathrm{mL}^{-1}$; mica; SFA ${ }^{33}$ & & 0.9 & & \\
$0.005 \mathrm{mg} \mathrm{mL}^{-1}$; silicon oxide; FTIR & 33 & 1.1 & 1.7 & 2.0 \\
$0.4 \mathrm{mg} \mathrm{mL}^{-1}$; mica; SFA (this work) & 1.4 & 0.9 & 4.0 & 1.8 \\
$1.0 \mathrm{mg} \mathrm{mL}^{-1}$; mica; SFA & & 1.5 & 2.1 & $2.6(0.5 \mathrm{M} \mathrm{NaCl})$
\end{tabular}

mica at these conditions. The adsorbed amount was generally slightly larger on silicon oxide than on mica. This is consistent with earlier findings that the limiting density of charges on silicon oxide is higher so that adsorption of polyelectrolyte is accompanied by additional ionization of $\mathrm{SiOH}$ groups to increase the charge density of the silicon oxide from an estimated $2 \times$ $10^{18} \mathrm{~m}^{-2}$ at $\mathrm{pH} 9.2$ and low salt concentration. ${ }^{18}$ At this $\mathrm{pH}$, the mica has already reached ${ }^{17,41,42}$ its maximum possible surface charge density, $2.1 \times 10^{18} \mathrm{~m}^{-2} \cdot{ }^{43}$

It is apparent from the data in Table 1 that (i) at low concentration of salt, the mass adsorbed of 14\% QPVP was approximately 1.5 times larger than the one of $98 \%$ QPVP, and (ii) the mass adsorbed of $98 \%$ QPVP increased with the salt concentration. The latter observation is consistent with screening of charges on the polyelectrolyte chain, causing less repulsion between segments. Regarding the first point, it is known that the amount of adsorbed polyelectrolyte at low electrolyte concentration decreases with increasing charge density. ${ }^{25,44}$ Comparison of SFA studies of $1 \%,{ }^{14} 10 \%,{ }^{13}$ and $30 \%{ }^{16}$ charged copolymer systems shows that the compressed film thickness (hard-wall) decreased from about $75 \AA$ to $40 \AA$ (for polyelectrolyte concentrations in the range $0.05 \mathrm{mg} \mathrm{mL}^{-1}$ to $0.2 \mathrm{mg}$ $\mathrm{mL}^{-1}$ ), and for the corresponding fully charged polyelectrolytes $^{9,10,39}$ the hard-wall was found at a film thickness of only $10 \AA$ (at concentrations from ${ }^{9} 0.01 \mathrm{mg} \mathrm{mL}^{-1}$ to $^{10} 0.1 \mathrm{mg} \mathrm{mL}^{-1}$ ). In a study of $76 \%$ quaternized poly-2-vinylpyridine (concentration $\left.0.1 \mathrm{mg} \mathrm{mL}^{-1}\right),{ }^{20}$ a hard-wall separation of $23 \AA$ was found at low $\mathrm{pH}$, where also the nonquaternized segments are charged, as compared to $33 \AA$ at high $\mathrm{pH}$. A similar trend is seen when comparing the adsorbed amounts of our 14\% and 98\% QPVP from buffer solution (cf. Table 1). This is consistent with an electrostatic mechanism of adsorption, where the number of charged units, not the total mass adsorbed, is the more important parameter.

The adsorbed amount gives information on the compensation of charges on the solid surface. In studies on highly charged cationic polyelectrolytes at lower concentration than in our experiment, it has been shown that the small counterions located at a mica surface in aqueous solution (neutralizing most but not all of its charge) are exchanged by entropically favored adsorbing charged polyelectrolyte segments so that the electrostatic repulsion between two surfaces disappears ${ }^{5,9,10,39}$ at a polyelectrolyte concentration where the total charge of the (flat) polyelectrolyte layer neutralizes approximately $80 \% \%^{9,10,39}$ of the maximum negative charge on the mica surface $\left(2.1 \times 10^{18}\right.$ charges $/ \mathrm{m}^{2}$ ). Based on the estimate of the adsorbed amount of $14 \%$ QPVP at strong compression $\left(1.4 \mathrm{mg} / \mathrm{m}^{2}\right.$, cf. Table 1), the total charge of the polyelectrolyte layer on each surface in this system is at most $1.0 \times 10^{18} \mathrm{~m}^{-2}$, and the surface charge of the mica is thus likely undercompensated. A similar conclusion has been drawn by Rojas et al., ${ }^{14}$ who found that the amount of charged segments in the adsorbed layer of a $1 \%$ charged cationic copolymer was lower than the surface charge of mica and silica at the conditions studied. The limitation in the adsorbed amount was ascribed to steric hindrance of charged segments reaching the solid surface through the extended layer of uncharged segments. ${ }^{14}$ These observations are in good agreement with FTIR measurements on 14\% QPVP adsorbed on silicon oxide from a buffer solution at $\mathrm{pH}=10.5,{ }^{18}$ which showed that a lower adsorbed amount (by almost a factor 2) of charged segments was obtained for this system than for $48 \%$ and $98 \%$ QPVP at similar conditions.

Following similar arguments, the charge in a compressed, adsorbed layer of $98 \%$ QPVP adsorbed from buffer is around $2.5 \times 10^{18} \mathrm{~m}^{-2}$, suggesting that the surface charge of the mica is overcompensated. This agrees well with theoretical predictions ${ }^{24}$ for fully charged polyelectrolyte at low salt concentration, and with experimental observations on $76 \%$ quaternized poly2-vinylpyridine at varying $\mathrm{pH}$ and a concentration of $0.1 \mathrm{mg}$ $\mathrm{mL}^{-1} .{ }^{20}$ Marra and Hair ${ }^{5}$ have shown that at low $\mathrm{pH}$, where the mica surface potential was about half of that in our system, approximately $70 \%$ protonated (nonquaternized) poly-2-vinylpyridine layers with a compressed (hard-wall) thickness of $12 \AA$ (i.e., an adsorbed amount of $0.7 \mathrm{mg} / \mathrm{m}^{2}$ ) caused charge reversal of the surfaces.

Static and Dynamic Forces as a Function of Separation. The static forces (normal to the substrates) as a function of separation distance were measured together with the corresponding elastic and viscous forces resisting oscillatory lateral shear. These forces are presented normalized by the radius of curvature of the surfaces, $R$, to allow comparison between different experiments. In all systems we studied, a repulsive static force was observed as the surfaces approached one another. We will see that this force contains an electrostatic component arising from the under- or overcompensation of the surface charge, as discussed above. At small separations, an attractive force appeared only between layers adsorbed at low electrolyte concentration, which will be discussed in more detail below. In systems where no adhesion was found, the static forces on separation were slightly weaker and had a shorter range than on approach, but recovered within the time the surfaces where held separated (about $10 \mathrm{~min}$ ) before the next approach. In all systems, reproducible force measurements were obtained on subsequent approaches unless a very high pressure had been applied. Hysteresis between purely repulsive forces measured on approach and separation has also been seen in other systems, particularly in the presence of a high solution concentration of polyelectrolyte, ${ }^{14,16}$ and in some cases a force dependent on the number of approaches, $1,2,5,7,9,11,20$ which we did not observe in our systems.

Forces between 14\% QPVP Layers Adsorbed from 1 mM Buffer. In the case of 14\% QPVP adsorbed from $1 \mathrm{mM}$ borate buffer with no added salt (Figure 1), the static repulsion between two layers (open symbols) had a considerably longer range than the shear forces. The interactions in Figure 1a, where the layers were not strongly compressed, were reproducible on subsequent approaches and separations. After strong compression to $F / R$ $>30 \mathrm{mN} / \mathrm{m}$, the range of the shear forces was reduced from about $200 \AA$ to $100 \AA$ (Figure 1b), indicating a flattening of the two layers of adsorbed polymer (irreversible on the timescale of our experiments). The magnitude and range of the static force was, however, reduced only slightly from the one in Figure 


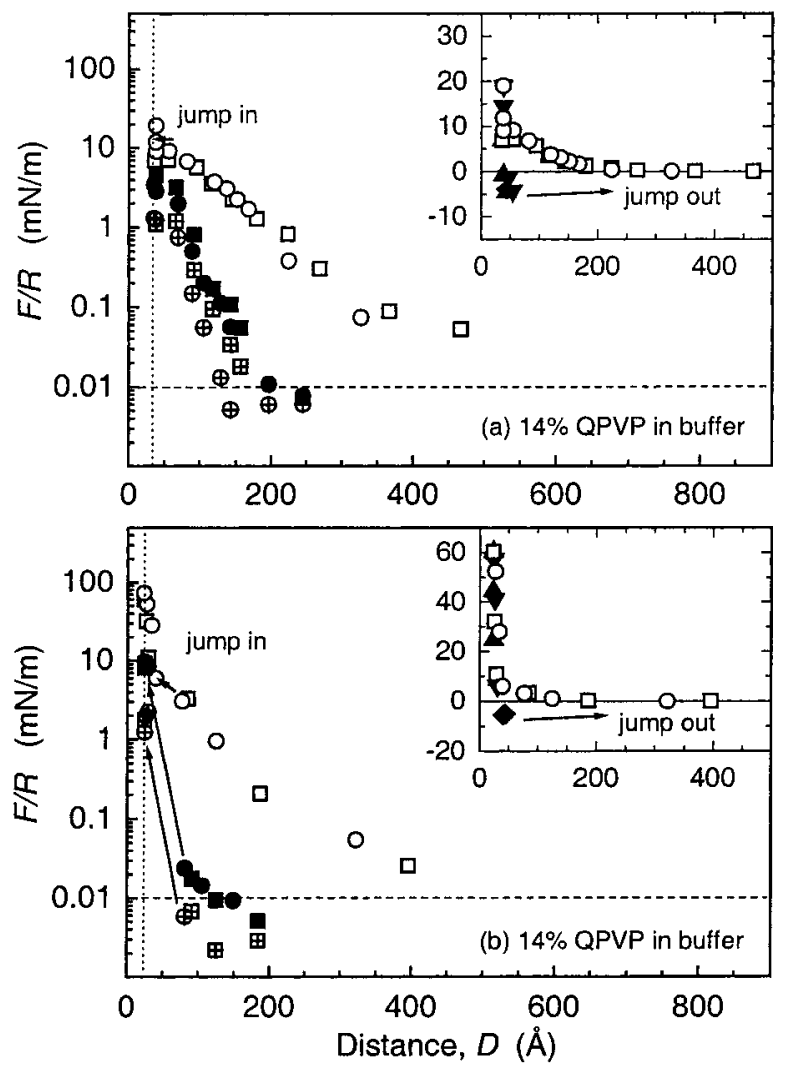

Figure 1. Static and dynamic forces between two opposed adsorbed layers of $14 \%$ quaternized poly-4-vinylpyridine (QPVP) in $1 \mathrm{mM}$ borate buffer $(\mathrm{pH}=9.2)$ as a function of separation between the mica substrates, D. In Figures 1, 4, and 7, each main panel shows static forces on compression (open symbols) together with the elastic (filled symbols) and viscous forces (cross-filled symbols) resisting oscillatory lateral shear at $f=1.3 \mathrm{~Hz}$ and a displacement amplitude of $x=10 \AA$. The forces were normalized by the radius of curvature, $R$. The dashed horizontal line indicates the sensitivity of the static force measurement. The insets show static forces during compression (open symbols) and separation (filled symbols) on linear scales. Panel (a): Low compression. Static repulsion was observed at $D<500 \AA$, followed by a jump in from $D \approx 55 \AA$ to $36 \AA$ (dotted vertical line). Upon separation, a jump out occurred from $D \approx 45-50 \AA$ and $F / R=-4 \mathrm{mN} / \mathrm{m}$. Shear forces could be detected at $D<200 \AA$. If at any point the polyelectrolyte layers were compressed strongly $(F / R>+30 \mathrm{mN} / \mathrm{m})$, the range of the shear interactions in all subsequent compressions was considerably reduced. The interaction forces between such irreversibly compressed layers are shown in panel (b). The range of the static repulsion then remained similar to that in panel (a), but the jump-in now occurred from $D=80 \AA$ to $35 \AA$ (hard-wall thickness ca. $25 \AA$ ) and the jump-out from $D=40-44 \AA$ at $F / R=-5 \mathrm{mN} / \mathrm{m}$.

1a. At this low electrolyte concentration, the static force is a combination of steric and electrostatic interactions, whereas the shear forces result from interactions between polymer chains. We note the reasonable agreement between the measured decay length, $67 \AA$, of the force at $D>150 \AA$ in Figure $1 \mathrm{~b}$ and the Debye length of $\kappa^{-1}=56 \AA$ in $1 \mathrm{mM} \mathrm{1:2} \mathrm{electrolyte,} \mathrm{or} \kappa^{-1}=$ $68 \AA$ in $2 \mathrm{mM} \mathrm{1:1}$ electrolyte ${ }^{32}$ (the limiting cases of no hydrolysis or complete hydrolysis of the borate ion). This comparison suggests that the long range part (or tail) of the static force in the 14\% QPVP system is of electrostatic origin and the actual extension of each layer is only $75-100 \AA$ from each surface in a noncompressed conformation, as detected from the onset of shear interactions in Figure 1a. Further analysis of the electrostatic force in Figure 1 is complicated by uncertainty about the distribution of the charge within the QPVP layer (causing possible shifts of the static force along the distance scale but not affecting the decay length. ${ }^{23}$ ). Compared to Figure 1b, the static force in Figure 1a has a longer decay length, 76 $\AA$, showing the influence of steric repulsion from the extended chains. Such discrepancies between the measured force and the expected Debye length, ${ }^{10,39}$ as well as a nonexponentially decaying force, ${ }^{13}$ have been found in other polyelectrolyte systems at increased concentration and have also been attributed to steric interactions in addition to electrostatic.

The observation of the range of steric interaction is supported by a study of Rojas et al. ${ }^{14}$ on a $1 \%$ charged cationic copolymer that was found to form brush-like layers (loops) containing a total amount of charged segments lower than the surface charge of mica and silica at the conditions studied. Following the approach in ref 14, the noncompressed layer on each surface in our system would contain tails and loops with a length of on average 45 segments ( $14 \%$ of $n=325$ ) with a possible extension of ca. 140 and $70 \AA$ from each surface, respectively, which is within the range estimated from the shear forces measured between two surfaces in Figure 1a. A similar analysis has been done for forces measured between thin gelatin layers adsorbed on mica, ${ }^{7}$ where a brush model for a steplike segment density profile superimposed on an exponentially decaying (electrostatic) force was used to fit the data.

Adhesion and Shear of Compressed 14\% QPVP Layers. In several studies on highly charged polymers at low concentration, ${ }^{5,9,10,20}$ adsorbing as nearly flat layers, it has been found that bridging of chains from one surface to the other gives rise to a long-range attraction on approach (at $D \leq 150 \AA$ ) and strong adhesion $\left((F / R)_{\text {adh }} \approx-20\right.$ to $\left.-100 \mathrm{mN} / \mathrm{m}\right)$. For $14 \%$ QPVP, which adsorbed in an extended conformation, we observed that the moderate range of the attraction (distance from which the surfaces jumped in) increased from $55 \AA$ (Figure 1a) to $80 \AA$ (Figure 1b) upon flattening of the adsorbed layer, while the magnitude of the adhesion on separation remained around -5 $\mathrm{mN} / \mathrm{m}$ (insets in Figures 1a and $1 \mathrm{~b}$ ). This increase in range cannot be explained by bridging on the approach, since bridging would be reduced after denser, less penetrable layers with fewer extending chains were formed during strong compression. The relatively low adsorbed amount of charged segments in this system (discussed previously) already indicated that the surfaces were not accessible to incoming chains even when the adsorbed layers were not compressed. Because some expansion of the layers was seen on separation, a bridging or entanglement developing in contact should not be excluded entirely, but the onset of the attractive force on approach of flattened layers is more consistent with van der Waals interactions.

The contribution of this attraction to the adhesion on separation can be estimated from van der Waals-Lifshitz theory ${ }^{38}$ by considering the hypothetical situation of compact (flat) layers of QPVP interacting across water. Tentatively, we assume the dielectric constant $\epsilon_{\mathrm{QPVP}}=3$ and refractive index $n_{\mathrm{QPVP}}=1.6$, giving $F / R=-3 \mathrm{mN} / \mathrm{m}$ at a separation of $10 \AA$ from contact (corresponding to the jump-out distance of $D \approx$ $45 \AA$ in Figure 1a, where the hard wall was at $36 \AA$ ). We suggest that the layer expansion seen as the compressive force was released arose from initially extended chains in trapped conformations. These chains prevented the more compact part of the QPVP layers (located directly at the surfaces) from coming in direct contact, which would be seen as a stronger adhesion due to van der Waals forces acting at a smaller separation.

The shear properties of the adsorbed layers were also investigated at large compression (the hard-wall separation) as a function of shear frequency and amplitude. Figure 2 shows the elastic and viscous moduli in the 14\% QPVP system at film thicknesses of $D=24$ and $33 \AA$. The response is dominated by 


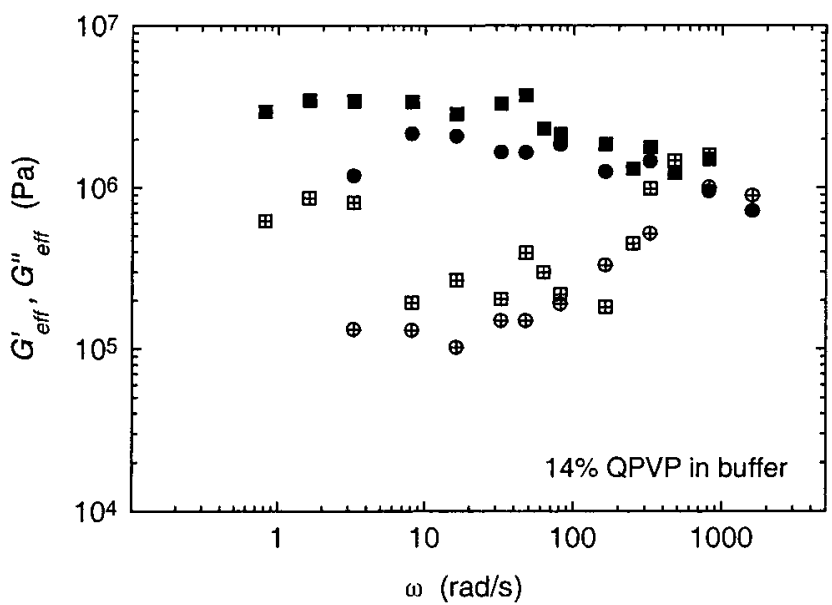

Figure 2. Responses to lateral shear at different frequencies of two opposed 14\% QPVP layers in $1 \mathrm{mM}$ borate buffer, at a total film thickness of $D=24 \AA$ (squares) and $33 \AA$ (circles). The effective elastic (filled symbols) and viscous moduli (cross-filled symbols) measured in oscillatory deformation with an amplitude of a few angstroms are plotted as functions of angular frequency $\omega(\omega=2 \pi f, f=0.13-130$ $\mathrm{Hz}$ ).

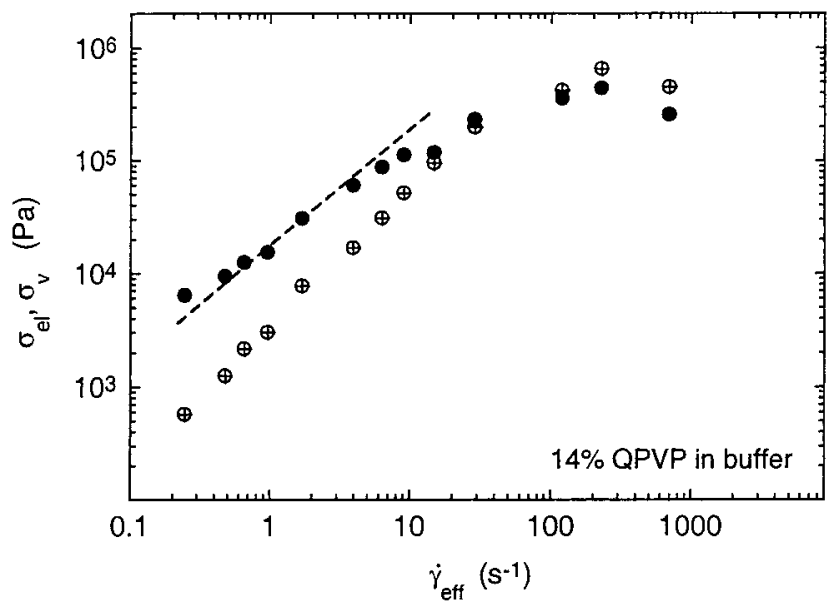

Figure 3. Responses to increasing displacement amplitude at $f=13$ $\mathrm{Hz}(\omega=82 \mathrm{rad} / \mathrm{s})$. Elastic (filled symbols) and viscous stress (crossfilled symbols) as a function of effective shear rate (strain $\times$ angular frequency) for 14\% QPVP, adsorbed as for Figures 1 and 2, measured at $D=34 \AA$. The critical strain for onset of nonlinear response (commencement of sliding) was approximately 0.5 . In the regime of linear response, a dashed line with a slope of 1 is drawn to guide the eye.

the elastic component, as is commonly observed for thin, confined polymer films where each molecule has many points of contact with the solid surface. ${ }^{45,46}$

The response of the same strongly compressed polymer layers to shear of increasing amplitude at a frequency of $f=13 \mathrm{~Hz}$ is shown in Figure 3 as elastic and viscous shear stress vs shear rate (strain $\times$ angular frequency). In this system and also for the 98\% QPVP discussed below, the transition from linear to nonlinear response (the commencement of sliding) was continuous. For 14\% QPVP it occurred at an effective strain (deflection amplitude divided by film thickness) of 0.5 .

Force and Adhesion Measurements on $98 \%$ QPVP Layers at Different Salt Concentrations. The forces between $98 \%$ QPVP layers adsorbed at different conditions are shown in Figure 4. In all cases, the range of the shear interactions in the 98\% QPVP systems was less sensitive to strong compression than the 14\% QPVP discussed above. Adsorption from a solution with higher electrolyte concentration resulted in a
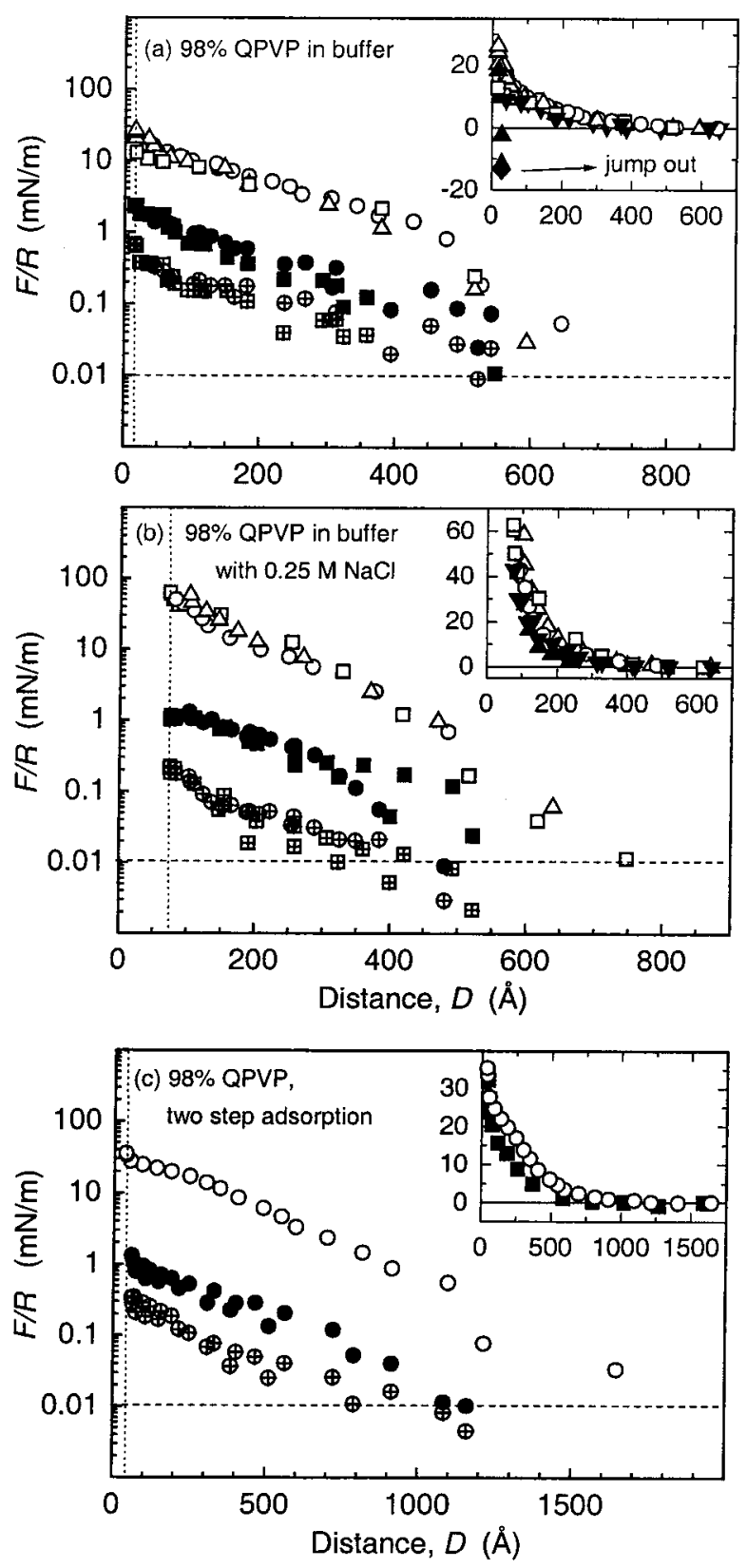

Figure 4. Contrast between one-step and two-step adsorption. Static and dynamic $(f=1.3 \mathrm{~Hz}$, displacement amplitude $x=10 \AA)$ forces between two opposed $98 \%$ QPVP layers adsorbed (a) from $1 \mathrm{mM}$ borate buffer; (b) from $1 \mathrm{mM}$ borate buffer containing $0.25 \mathrm{M} \mathrm{NaCl}$; and (c) by $1 \mathrm{~h}$ adsorption from $1 \mathrm{mM}$ borate buffer, then $1 \mathrm{~h}$ adsorption from $1 \mathrm{mM}$ borate buffer containing $0.25 \mathrm{M} \mathrm{NaCl}$ (two-step adsorption). Symbols and insets as defined in Figure 1. Hard-wall repulsion between strongly compressed layers was observed at (a) $D=16 \AA$, (b) 70-80 $\AA$, and (c) 35-45 $\AA$. Note the different scale on the distance axis in panel (c). Inset in panel (a): After strong compression to $F / R>+20$ $\mathrm{mN} / \mathrm{m}$, a jump out was observed from $D=28 \AA$ and $F / R=-13$ $\mathrm{mN} / \mathrm{m}$, but if the compression was lower, the repulsive forces were reversible, as shown by $(\boldsymbol{\nabla})$ symbols. No adhesion was found for the systems in panels (b) and (c), where the insets show the static forces on linear scales.

significantly larger adsorbed amount (Table 1) as judged from the hard-wall film thickness measured on compression. The origin of the interactions observed at different conditions is discussed below.

(a) 98\% QPVP in $1 \mathrm{mM}$ Buffer. When adsorbed from $1 \mathrm{mM}$ borate (Figure 4a), the compressed (hard-wall) thickness of two 98\% QPVP layers was only $16 \AA$, but in contrast to $14 \%$ QPVP (Figure 1a), the distance ranges of the static force and the shear 
forces were considerably larger and nearly coincided. As mentioned previously, an electrostatic repulsion is likely to be present in this system due to the charge reversal. In this situation the adsorbed amount is limited because of a repulsive (positive) potential building up as the surface charge on the mica is being overcompensated. However, the measured static force in our system did not decay exponentially at large $D$. At smaller separations the decay length was ca. $78 \AA$ (to be compared to $\kappa^{-1}=56$ or $67 \AA$, as discussed for $14 \%$ QPVP in buffer), which together with the shear data suggest that the static force also at large separations is influenced by steric repulsion between chains that are strongly stretched due to their high charge. An adsorbed amount similar to the one in our system was found in experiments where the $98 \%$ QPVP was allowed to adsorb onto mica from a $0.005 \mathrm{mg} \mathrm{mL}^{-1}$ solution in $1 \mathrm{mM}$ borate buffer. ${ }^{33}$ At this concentration, the long-range interactions were well described as purely electrostatic (decay length $60 \AA$ ) ), suggesting that the chains adsorbed in a flat conformation. At very high concentration, $1 \mathrm{mg} \mathrm{mL}-1$, a larger adsorbed amount was obtained (cf. Table 1), ${ }^{33}$ but in this case the observed decay length of the force, $30 \AA$, was shorter than the expected Debye length (taking into account the ionic strength of the polyelectrolyte in the adsorption solution kept between the surfaces in the experiments ${ }^{33}$ ).

This aspect of our results is qualitatively similar to experiments by Dahlgren et al. on another fully quaternized polyelectrolyte adsorbed at low ionic strength at concentrations lower than in our experiment $\left(0.05-0.1 \mathrm{mg} \mathrm{mL}^{-1}\right)$, but high enough to cause overcompensation of the surface charge. ${ }^{10}$ Similarly to adsorption at even lower concentration $\left(0.01 \mathrm{mg} \mathrm{mL}^{-1}\right)$, the compressed layer thickness in that system was $\leq 10 \AA$, suggesting a small adsorbed amount, but at the higher concentrations, repulsive forces were found with a longer decay length than the Debye length. ${ }^{10}$ The longer decay length was interpreted as chains extending into solution to add a steric force to the electrostatic. ${ }^{10}$ Our data on the shear forces (Figure 4a) confirm this result and show that at higher adsorbed amounts, this extension can be large due to the strong stretching of highly charged chains. The larger hard-wall thickness in our system, $16 \AA$, is due to a larger adsorbed amount at higher concentration.

In this system, no jump-in was seen on compression, and an adhesive force with a magnitude of ca. $-13 \mathrm{mN} / \mathrm{m}$ at the jumpout from $D=28 \AA$ developed only after applying a compressive force larger than $+20 \mathrm{mN} / \mathrm{m}$ at the hard-wall separation. When the compressive force was lower, the separation was reversible and resembled the curve obtained on approach, as shown in the inset in Figure 4a (filled triangles pointing down). The adhesion likely comes from a combination of bridging and van der Waals forces, of which the latter contribute approximately $-3 \mathrm{mN} / \mathrm{m}$ at a separation of $10 \AA$ from contact in a system modeled as flat layers, as discussed above for $14 \%$ QPVP. The adsorbed amount in our system, $0.9 \mathrm{mg} / \mathrm{m}^{2}$, would correspond to one complete (compact), flat monolayer on each surface as estimated by Marra and Hair for poly-2-vinylpyridine. ${ }^{5}$ However, at the conditions in our experiment, the layer is not flat but some of the chains are clearly extending into solution at low compression. Since the adhesion did not develop until after strong compression, a rearrangement must occur of the trapped conformation of the extended chains that otherwise keep the layers separated at a particular distance. Rearrangement leading to bridging attraction appearing only after adhesive contact has also been proposed for highly protonated poly-2-vinylpyridine on mica at a lower surface coverage than in our system. ${ }^{5}$ Entanglement between chains adsorbed to different surfaces is also conceivable but is expected to be low because highly charged chains repel one another.

(b) $98 \%$ QPVP in Buffer with Added $0.25 \mathrm{M} \mathrm{NaCl}$. If the adsorption of $98 \%$ QPVP was allowed from borate buffer containing $0.25 \mathrm{M} \mathrm{NaCl}$ (Figure 4b), the range of interaction was similar to the case without salt (Figure 4a), but the hardwall thickness and the inferred adsorbed amount (Table 1) were significantly larger. No adhesion was observed in this system even after applying large compressive forces (inset in Figure 4b). Compared to the case of $98 \%$ QPVP adsorbed at low electrolyte concentration (Figure 4a), the extension of the shear forces was not quite as long as the static forces. In this system, this is not because of additional electrostatic forces as for $14 \%$ QPVP (Figure 1a), since the range of these should be short at high ionic strength, but is likely due to the different conformation of the adsorbed QPVP. The conformation and adsorbed amount are the result of screening of charges on the polyelectrolyte chain (causing less repulsion between segments, and thus a conformation more similar to a neutral polymer chain), ${ }^{21-25}$ and of adsorption of small cations to the surfaces. The screening of the charge on the pyridinium ion is dependent on the counterion, and decreases in the order $\mathrm{I}^{-}>\mathrm{Br}^{-}>\mathrm{Cl}^{-} .47-50$ As an example, it can be inferred that at an $\mathrm{I}^{-}$concentration of $0.3 \mathrm{M}$, more than $40 \%$ of the charges on free QPVP chains are screened. ${ }^{47,50}$

At low electrolyte concentration (Figure 4a), the adsorbed amount was low, and the chains extending from the surfaces were strongly stretched. In $0.25 \mathrm{M} \mathrm{NaCl}$ (Figure 4b), a larger fraction formed loops and a small amount of the polymer was found in long tails, ${ }^{24}$ a conformation resembling the one expected for adsorbed uncharged polymer. As a result of the lower stretching, the range of the static force did not increase in proportion to the adsorbed amount, as may be seen by comparing Figures $4 \mathrm{a}$ and $4 \mathrm{~b}$. A change in conformation from flat layers to "loops" or extended layers when increasing the salt concentration has been observed in several systems of polyelectrolytes adsorbed from lower concentration onto mica. ${ }^{5,7,9}$ This is supported by measurements of the infrared dichroism of the pyridinium ring of our $98 \%$ QPVP on silicon oxide, where the pyridinium segments were preferentially oriented parallel to the surface at low salt concentration, and a more isotropic orientation was found in $0.25 \mathrm{M} \mathrm{NaCl} .^{18}$

(c) Two-step Adsorption of $98 \%$ QPVP. The effect of adsorption history is shown in Figure 4c. The polymer was allowed to adsorb in two steps (procedures that, when done separately, result in the interactions shown in Figures $4 \mathrm{a}$ and 4b). In this case the extension of the forces was significantly larger than obtained separately for the two adsorption conditions (note the different distance scale in Figure 4c), even though the adsorbed amount was only twice the one obtained in the first step (Table 1), and the magnitude of the force at smaller separations did not exceed the one in Figure 4a. This is consistent with FTIR experiments and previous force measurements ${ }^{33}$ that suggest that the polymer chains adsorbing during a second adsorption step to the already partially covered surfaces have fewer contact points with the solid surface per chain and thus dangle far out into the solution to give a large increase in the range of the forces. The measured static force range suggests that some chains extend about $750 \AA$ from each surface, which would be close to the contour length of a chain with $n=325$. However, we note that the polydispersity of the QPVP is 1.23, which implies that also significantly longer chains are present in the system, and the extending chains are therefore not necessarily fully stretched. The possible preferential adsorption 

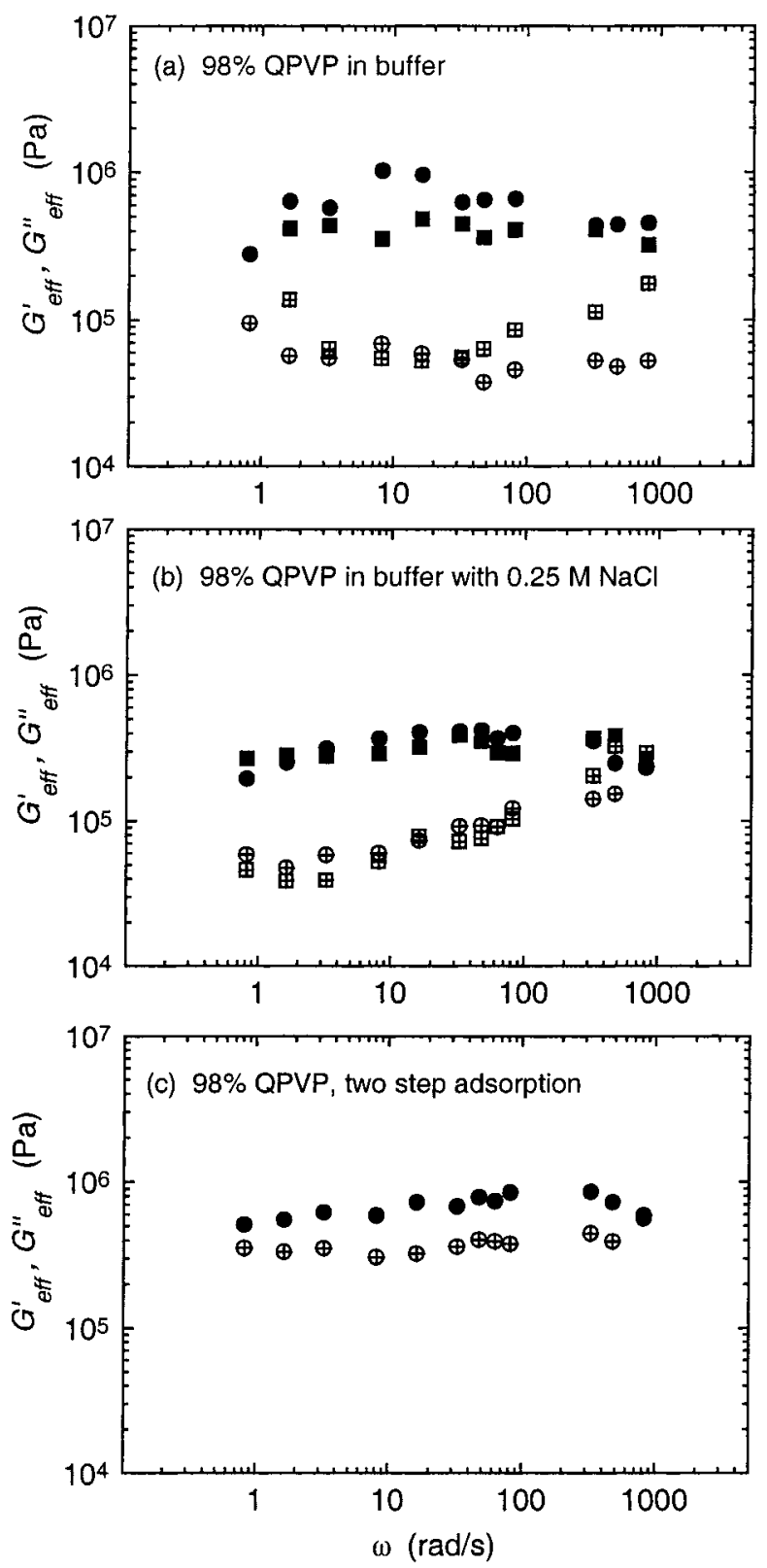

Figure 5. Responses to lateral shear at different frequencies of compressed 98\% QPVP films adsorbed at the same conditions as in Figure 4: (a) from $1 \mathrm{mM}$ borate buffer, $D=16 \AA$; (b) from $1 \mathrm{mM}$ borate buffer with added $0.25 \mathrm{M} \mathrm{NaCl}, D=76$ and $83 \AA$ (circles and squares, respectively); and (c) by $1 \mathrm{~h}$ adsorption from $1 \mathrm{mM}$ borate buffer, then $1 \mathrm{~h}$ adsorption from $1 \mathrm{mM}$ borate buffer with added 0.25 $\mathrm{M} \mathrm{NaCl}$ (two-step adsorption), $D=48 \AA$. The symbols are the same as in Figure 2.

of longer polyelectrolyte chains has not been investigated for our system, but has been discussed for $\mathrm{TiO}_{2}$ and $\mathrm{SiO}_{2}$ surfaces. ${ }^{12}$

A qualitatively similar increase in the range of the static force after increasing the salt concentration in a second step has also been observed at higher concentration $\left(1.0 \mathrm{mg} \mathrm{mL}^{-1}\right)$ of $98 \%$ QPVP. ${ }^{33}$ These results differ from observations on another cationic polyelectrolyte at significantly lower concentration, 0.02 $\mathrm{mg} \mathrm{mL}{ }^{-1}{ }^{11}$ where the extension of the forces was longer if adsorption was done directly from a higher electrolyte concentration than if it was done in two steps. It is likely that the different results arise from the differences in polyelectrolyte concentration in the adsorption solution. It is possible that the conformation obtained in our system was a nonequilibrium situation, although no changes could be seen on the time-scale of our experiments. A larger hysteresis on separation (inset in
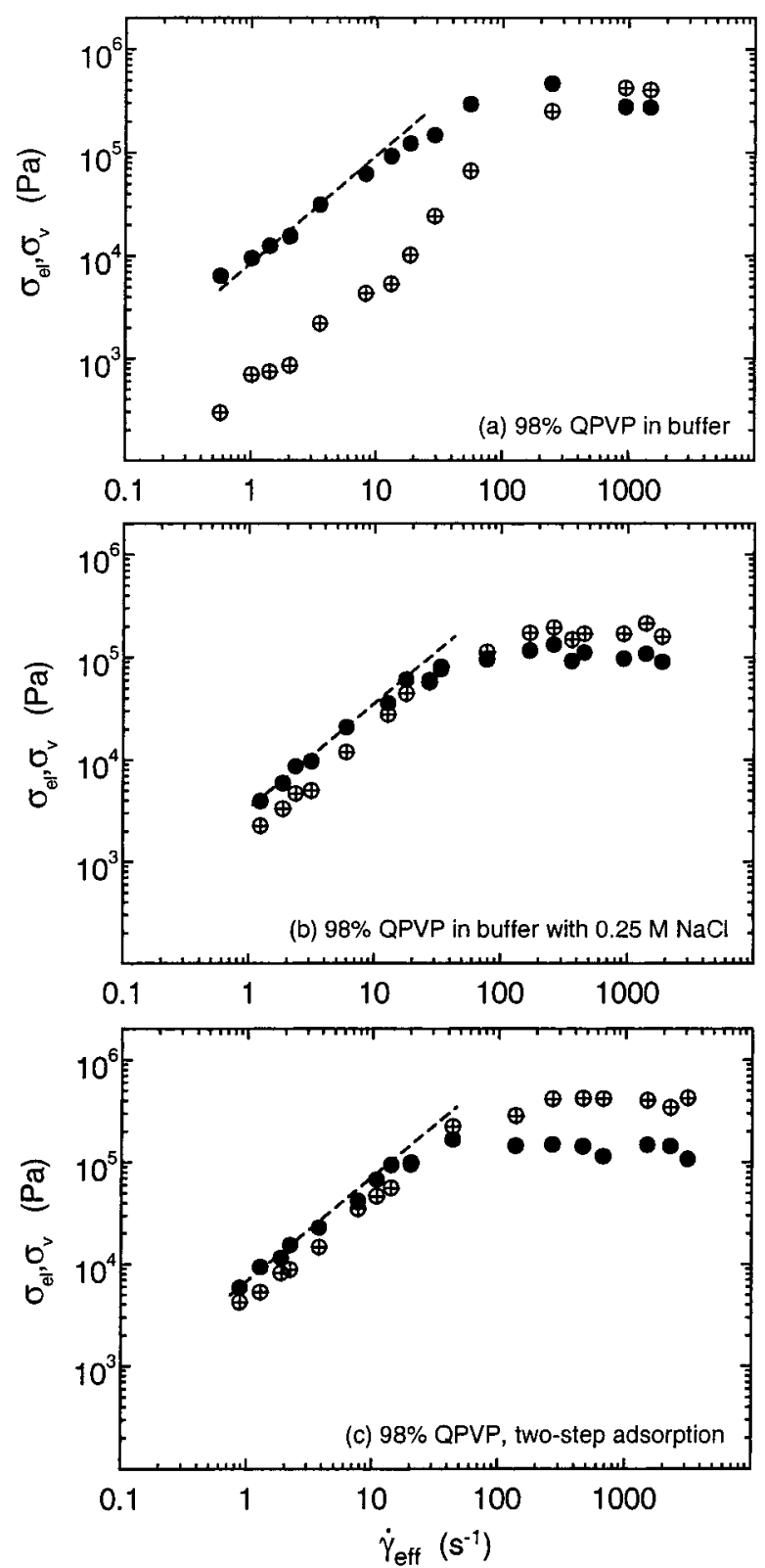

Figure 6. Responses to increasing displacement amplitude at $f=13$ $\mathrm{Hz}(\omega=82 \mathrm{rad} / \mathrm{s})$. Elastic (filled symbols) and viscous stress (crossfilled symbols) as a function of effective shear rate for 98\% QPVP adsorbed at the same conditions as for Figures 4 and 5: (a) from 1 $\mathrm{mM}$ borate buffer, $D=16 \AA$; (b) from $1 \mathrm{mM}$ borate buffer with added $0.25 \mathrm{M} \mathrm{NaCl}, D=83 \AA$; and (c) by $1 \mathrm{~h}$ adsorption from $1 \mathrm{mM}$ borate buffer, then $1 \mathrm{~h}$ adsorption from $1 \mathrm{mM}$ borate buffer with added 0.25 $\mathrm{M} \mathrm{NaCl}$ (two-step adsorption), $D=48 \AA$. The critical strain for onset of nonlinear response was $1-1.5,0.5$, and 0.6 , respectively, in panels (a) through (c). In the regime of linear response, a dashed line with a slope of 1 is drawn to guide the eye.

Figure 4c) than in the systems obtained by one-step adsorption (insets in Figures $4 \mathrm{a}$ and $4 \mathrm{~b}$ ) indicates higher sensitivity of this system to compression. No adhesion was seen between $98 \%$ QPVP layers after two-step adsorption, even though the hardwall thickness and the adsorbed amount inferred are very similar to the ones found for 14\% QPVP in Figure 1a. We suggest that the better filling of the surface sites obtained with 98\% QPVP and the conformation of the additional chains on compression prevented adhesive contact.

Shear Response of Confined Layers of $98 \%$ QPVP. A qualitatively similar response to small-amplitude oscillatory shear was observed for 98\% QPVP adsorbed from $1 \mathrm{mM}$ borate 


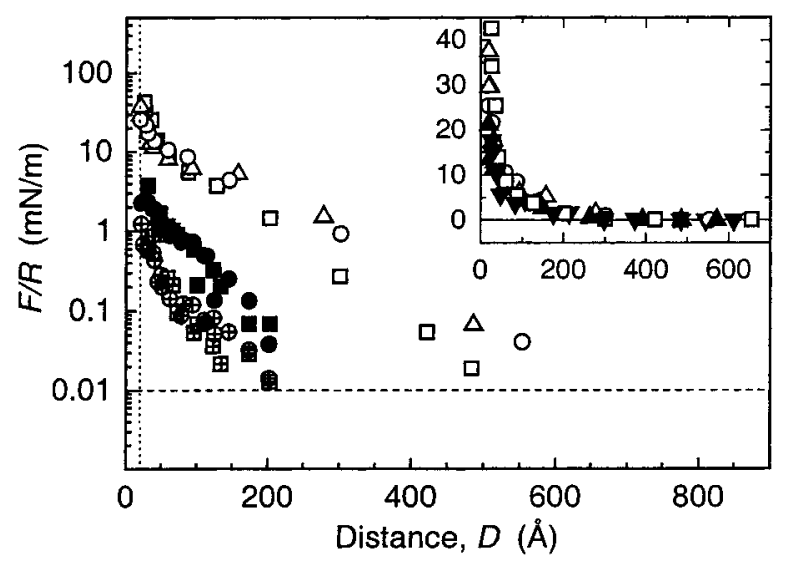

Figure 7. Asymmetric system. Static and dynamic $(f=1.3 \mathrm{~Hz}$, displacement amplitude $x=10 \AA$ ) forces between an adsorbed layer of $14 \%$ QPVP on one mica surface and an adsorbed layer of $98 \%$ QPVP on the other, in $1 \mathrm{mM}$ borate buffer. The symbols are the same as in Figure 1. The hard-wall thickness was $D=22 \AA$. The inset shows the static forces during compression (open symbols) and separation (filled symbols) on linear scales.

buffer (Figure 5a) as from buffer with $0.25 \mathrm{M} \mathrm{NaCl}$ (Figure $5 b)$. In both cases, the elastic component dominated. In buffer with $0.25 \mathrm{M} \mathrm{NaCl}$, the overall magnitude was somewhat lower (reflecting the larger film thickness) and the viscous response approached the magnitude of the elastic response at the highest frequency. However, in the system formed by two-step adsorption (Figure 5c), the viscous response was of similar magnitude as the elastic (smaller only by a factor 2) throughout the frequency range investigated. The behavior of this system is consistent with a different structure of the compressed adsorbed layer compared to the ones obtained in adsorption in one step, where a predominantly elastic response is caused by polymer chains having many contacts with the solid surface, which restricts their motion. In the structure obtained in the two-step adsorption, the compressed film contains a middle region of chains with fewer contacts with the solid surface and their motion can therefore be expected to be less restricted. When comparing the shear moduli of two compressed films with similar thickness, 14\% QPVP in $1 \mathrm{mM}$ buffer solution (Figure 2) and 98\% QPVP after two-step adsorption (Figure 5c), the proportions between the elastic and viscous components can be easily compared, again suggesting different structures.

The responses of the compressed 98\% QPVP layers to increasing displacement amplitude at a frequency of $f=13 \mathrm{~Hz}$ are shown in Figure 6. The limiting strain was 1-1.5, 0.5, and 0.6 for $98 \%$ QPVP adsorbed at different conditions as described in Figure 6a, b, and c, respectively. Generally, more mobile layers are expected to have larger limiting strain than solid- or rubber-like ones, which explains the value 0.6 for the two-step adsorption compared to 0.5 for the $14 \%$ QPVP film with similar thickness (Figure 3) and 0.5 for $98 \%$ QPVP in buffer with 0.25 $\mathrm{M} \mathrm{NaCl}$. The limiting strain of $1-1.5$ for $98 \%$ QPVP adsorbed from buffer (Figure 6a) is unexpected for molecules with many contacts with the surface, which would typically give a rubberlike response. As discussed previously, the magnitude of the adhesion in this system suggests that bridging occurred when the adsorbed layers were compressed strongly. A coupling of the surfaces by bridging chains might possibly explain this unusual shear behavior.

Interactions in an Asymmetric System of 14\% and $98 \%$ QPVP. In Figure 7, we show the interactions in an asymmetric system where one surface carried 14\% QPVP and the other $98 \%$ QPVP, both adsorbed from $1 \mathrm{mM}$ borate buffer. The interaction forces appear to be intermediate to the ones seen separately for symmetric systems (Figures 1a and 4a), as were the shear moduli and stress (not shown). No adhesion was observed even after strong compression. Bridging is not expected because of the steric hindrance at the $14 \%$ QPVP surface. As a point of speculation, we note the increasing slope of the repulsive interaction (static force) at short separations in this system. Possibly, the conformation of the compressed extended chains of the 98\% QPVP layer against the compact 14\% QPVP layer prevented the layers from coming sufficiently close to experience an observable van der Waals attraction.

\section{Conclusions}

In contrast to prior experiments on strong polyelectrolytes at very low solution concentrations, extended layers of both $14 \%$ and 98\% QPVP resulted from adsorption at the higher concentration $\left(0.4 \mathrm{mg} \mathrm{mL}^{-1}\right)$ studied here. At high degree of quaternization and low electrolyte concentration, the chains were strongly stretched. As predicted theoretically, the extension became more similar to that of neutral polymer when the ionic strength was high. Even after strong compression, the adsorbed layers remained extended after separation, which complicates the determination of the charge on the solid surface and thus the analysis of the electrostatic contribution to the repulsive force. The adsorbed amounts on mica were lower than on silicon oxide at the same $\mathrm{pH}$ and ionic strength, but qualitatively similar changes occurred upon changing the solution conditions. A nonequilibrium structure of the adsorbed layer, with a fraction of adsorbed chains extending far into solution, was formed after two-step adsorption.

Acknowledgment. We thank M. Gee for helpful discussions. This work was supported by the U.S. National Science Foundation, Grant CMS-98-12941.

\section{References and Notes}

(1) Cafe, M. C.; Robb, I. D. J. Colloid Interface Sci. 1982, 86, 411. 80,865 .

(2) Luckham, P. F.; Klein, J. J. Chem. Soc., Faraday Trans. 1 1984,

(3) Cosgrove, T.; Obey, T. M.; Vincent, B. J. Colloid Interface Sci. 1986, $111,409$.

(4) Durand-Piana, G.; Lafuma, F.; Audebert, R. J. Colloid Interface Sci. 1987, 119, 474

(5) Marra, J.; Hair, M. L. J. Phys. Chem. 1988, 92, 6044.

(6) Kawanishi, N.; Christenson, H. K.; Ninham, B. W. J. Phys. Chem. 1990, $94,4611$.

(7) Kamiyama, Y.; Israelachvili, J. Macromolecules 1992, 25, 5081.

(8) Claesson, P. M.; Ninham, B. W. Langmuir 1992, 8, 1406.

(9) Dahlgren, M. A. G.; Waltermo, A.; Blomberg, E.; Claesson, P. M.; Sjöström, L.; Åkesson, T.; Jönsson, B. J. Phys. Chem. 1993, 97, 11769.

(10) Dahlgren, M. A. G.; Claesson, P. M.; Audebert, R. J. Colloid Interface Sci. 1994, 166, 343.

(11) Dahlgren, M. A. G.; Hollenberg, H. C. M.; Claesson, P. M. Langmuir 1995, 11, 4480.

(12) Hoogeveen, N. G.; Cohen Stuart, M. A.; Fleer, G. J. J. Colloid Interface Sci. 1996, 182, 133. Ibid. p 146.

(13) Kjellin, U. R. M.; Claesson, P. M.; Audebert, R. J. Colloid Interface Sci. 1997, 190, 476

(14) Rojas, O. J.; Claesson, P. M.; Muller, D.; Neuman, R. D. J. Colloid Interface Sci. 1998, 205, 77.

(15) Lowack, K.; Helm, C. A. Macromolecules 1998, 31, 823.

(16) Claesson, P. M.; Fielden, M. L.; Dedinaite, A.; Brown, W.; Fundin, J. J. Phys. Chem. B 1998, 102, 1270.

(17) Hartley, P. G.; Scales, P. J. Langmuir 1998, 14, 6948.

(18) Sukhishvili, S. A.; Granick, S. J. Chem. Phys. 1998, 109, 6861.

(19) Sukhishvili, S. A.; Granick, S. J. Chem. Phys. 1998, 109, 6869.

(20) Maurdev, G.; Meagher, L.; Ennis, J.; Gee, M. L. Macromolecules, in press.

(21) Hesselink, F. Th. J. Colloid Interface Sci. 1977, 60, 448

(22) Van der Schee, H. A.; Lyklema, J. J. Phys. Chem. 1984, 88, 6661. 2461. 
(24) Böhmer, M. R.; Evers, O. A.; Scheutjens, J. M. H. M. Macromolecules 1990, 23, 2288.

(25) van de Steeg, H. G. M.; Cohen Stuart, M. A.; de Keizer, A.; Bijsterbosch, B. H. Langmuir 1992, 8, 2538, and references therein.

(26) Israëls, R.; Leermakers, F. A. M.; Fleer, G. J.; Zhulina, E. B. Macromolecules 1994, 27, 3249.

(27) Linse, P. Macromolecules 1996, 29, 326.

(28) Fleer, G. J. Ber. Bunsen-Ges. Phys. Chem. 1996, 100, 936

(29) Châtellier, X.; Joanny, J.-F. J. Phys. II (France) 1996, 6, 1669.

(30) Netz, R. R.; Joanny, J.-F. Macromolecules 1999, 32, 9013. Ibid. p 9026.

(31) Borukhov, I.; Andelman, D.; Orland, H. J. Phys. Chem. B 1999, 103,5042 .

(32) Hiemenz, P. C. Principles of Colloid and Surface Chemistry, 2nd ed.; Marcel Dekker: New York, 1986.

(33) Sukhishvili, S. A.; Dhinojwala, A.; Granick, S. Langmuir 1999 15,8474 .

(34) Israelachvili, J. N.; Adams, G. E. J. Chem. Soc., Faraday Trans. 1 1978, 74, 975

(35) Peachey, J.; Van Alsten, J.; Granick, S. Rev. Sci. Instrum. 1991, $62,463$.

(36) Granick, S.; Hu, H.-W. Langmuir 1994, 10, 3857.
(37) Israelachvili, J. N. J. Colloid Interface Sci. 1973, 44, 259.

(38) Israelachvili, J. N. Intermolecular and Surface Forces, 2nd ed; Academic Press: London, 1991.

(39) Dahlgren, M. A. G. Ph.D. Thesis, Royal Institute of Technology, Stockholm, Sweden, 1995.

(40) Polymer Handbook, 3rd ed.; Brandrup, J., Immergut, E. H., Eds.;

Wiley: New York, 1989.

(41) Scales, P. J.; Grieser, F.; Healy, T. W. Langmuir 1990, 6, 582

(42) Shubin, V. E.; Kekicheff, P. J. Colloid Interface Sci. 1993, 155, 108

(43) Gaines, G. L.; Tabor, D. Nature (London) 1956, 176, 1305.

(44) Böhmer M. R.; Heesterbeek, W. H. A.; Deratani, A.; Renard, E. Colloids Surf., A 1995, 99, 53.

(45) Hu, H.-W.; Granick, S. Science 1992, 258, 1339. Granick, S.; Hu, H.-W. Langmuir 1994, 10, 3857.

(46) Cho, Y.-K.; Granick, S. Wear 1996, 200, 346.

(47) Slough, W. J. Chem. Soc., Faraday Trans. 1959, 55, 1030.

(48) Südholter, E. J. R.; Engberts, J. B. N. J. Phys. Chem. 1979, 83, 1854

(49) Beer, M.; Schmidt, M.; Muthukumar, M. Macromolecules 1997 30,8375 .

(50) Biesalski, M., Ph.D. Thesis, University of Mainz, Germany, 1999. 